\title{
Investigation of Social Appearance Anxiety of Students of Faculty of Sport Sciences and Faculty of Education in Terms of Some Variables
}

\section{Tarik SEVINDI}

Aksaray University Faculty of Sport Sciences, Turkey.

Email:tariksevindi@gmail.com Tel:+905059043333

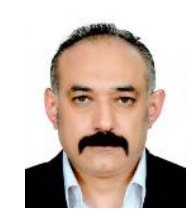

\begin{abstract}
This study was carried out to examine the social appearance anxiety of the faculty of sports sciences and faculty of education in terms of some variables. In this context, the social appearance anxiety scale was applied to the students of the sports sciences who received sports education and the students of the education faculty who did not receive sports education. Independent samples ttest, one-way ANOVA, and LSD tests were used to analyze the data. As a result of the analysis, the social appearance anxiety scale score was found 34.43 in women and 35.11 in men. On the other hand, the social appearance anxiety scale score was found to be 32.89 in students of faculty of sport sciences and 36.68 in students of faculty of education. There was no significant difference in social appearance anxiety scale scores in terms of gender variable ( $p>0.05)$. A significant difference was observed in the social appearance anxiety scale scores in terms of sport education status variable $(\mathrm{p}<0.001)$. The social appearance anxiety scores of the students who were satisfied with their own body weight and height were found lower than those who were not satisfied, which was found statistically significant $(\mathrm{p}<0.001)$. The results of this research showed that the social appearance anxiety of students of the faculty of sport sciences who receive sports education is lower than those of the faculty of education students who do not receive sports education.
\end{abstract}

Keywords: Social appearance anxiety, Students, Faculty of sport sciences, Faculty of education.

Citation | Tarik SEVINDI (2020). Investigation of Social Appearance Anxiety of Students of Faculty of Sport Sciences and Appearance Anxiety of Students of Faculty of Sport Sciences and
Faculty of Education in Terms of Some Variables. Asian Journal of Education and Training, 6(3): 541-545.

History:

Received: 15 June 2020

Revised: 17 July 2020

Accepted: 19 August 2020

Published: 3 September 2020

Licensed: This work is licensed under a Creative Commons

Licensed: This work is
Attribution 3.0 License (cc) Er

Publisher: Asian Online Journal Publishing Group

\section{Contents}

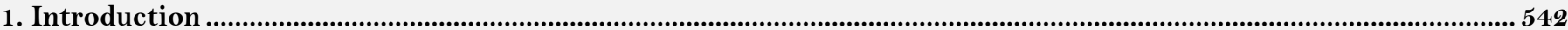

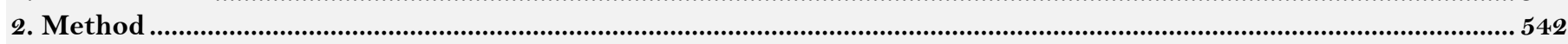

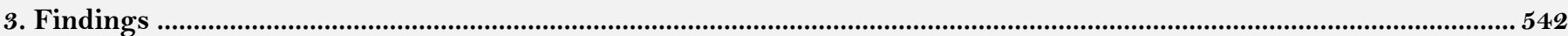

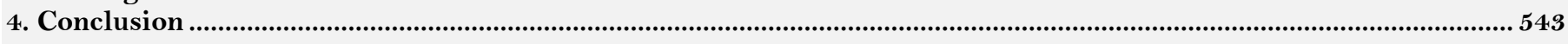

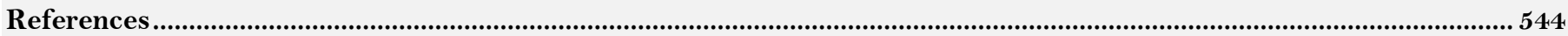

Competing Interests: The author declares that there are no conflicts of interests regarding the publication of this paper.

Transparency: The author confirms that the manuscript is an honest, accurate, and transparent account of the study was reported; that no vital features of the study have been omitted; and that any discrepancies from the Etudy as planned have been explained.

Ethical: This study follows all ethical practices during writing 


\section{Contribution of this paper to the literature}

This study contributes to existing literature by examining the social appearance anxiety of the faculty of sports sciences and faculty of education in terms of some variables.

\section{Introduction}

Individuals have their own perceptions of their physical structure, and these perceptions can give people morale in some cases, and in others, they can create anxiety. Social appearance anxiety is a phenomenon that occurs in the mind of the person due to physical structure. Social appearance anxiety is expressed as individuals' feelings towards the assessment of their physical structures by other individuals. Individuals are sometimes concerned about how other people look at themselves. If anxiety is high, individuals take action to leave a positive impression on their environment and if they fail, they may experience disappointment (Mülazımoğlu, Erturan, \& Arslan, 2014). Social appearance anxiety, which is also considered as social worry, can be expressed as the physical assessment of the individual (Yüceant, 2013). While individuals who evaluate themselves positively in terms of appearance, feel more successful and safe in interpersonal communication and relationships, individuals who do not like themselves physically feel constantly uneasy, unworthy and insecure in their lives (Ergür, 1996). Social appearance anxiety is a concept that includes physical features such as height, weight, and muscle structure on the one hand, and physical features such as skin color, nose, eye structure, and smile of the individual on the other hand (Hart et al., 2008). Depending on these features, social appearance anxiety has negative effects on individuals' academic, professional, and social aspects (Dindar \& Akbulut, 2015). University students tend to make a positive impression on other individuals with their physical appearance around the school. On the other hand, it is accepted as a possible situation that students who do not like themselves physically experience psychological problems (Kılıç, 2015). In general, physical activity brings individuals together regardless of religion, language, race, gender, age, and culture, and creates a positive communication by creating a personal and social identity and team spirit (Yilldiran \& Yetim, 1996). In addition, when individuals engage in physical activity, they gain a sense of self-expression and self-confidence, improving their skills of success and social communication. In addition, it reduces mental exhaustion and tension, and solidarity and gentleman's spirit emerges (Aksoy, Aslan, \& İmamoğlu, 2020; Gür \& Küçükoğlu, 1992).

It is a matter of curiosity to examine some variables on university students' perceptions of social appearance anxiety. Therefore, in this study, some variables were discussed on the students of the faculty of sports sciences and students of the faculty of education, and the answers to the research questions developed below were sought:

$R Q 1$. Do anthropometric properties of students differ in terms of gender variable?

$R Q^{2}$. Do the social appearance anxieties of students differ in terms of gender variable?

$R Q 3$. Do the social appearance anxieties of the students differ in terms of the variable of sports education status?

$R Q 4$. Do the social appearance anxieties of students differ in terms of body weight and height?

\section{Method}

\subsection{Sample Size}

A simple random sampling method was used in the study. Data were obtained from the Faculty of Sports Science and Faculty of Education student at a state university in Turkey. Students aged 18-25 years participated in the study voluntarily. As a result, 448 questionnaires that were filled in completely were evaluated.

\subsection{Measurement Instrument}

Social Appearance Anxiety Scale was developed by Hart et al. (2008) and later, Turkish validity and reliability studies were conducted by Doğan (2009). This scale, which measures the Social Appearance Anxiety of the individuals, consists of 16 items. The Social Appearance Anxiety Scale has a Likert-type scale ranging from 1 (not at all) to 5 (extremely). Examples of the statements are "I get nervous talking to people because of the way I look," and "I worry that others talk about flaws in my appearance when I'm not around." The first item of the scale is coded in reverse. High scores on the Social Appearance Anxiety scale indicate that individuals have high apprehension anxiety. In addition, the Cronbach's alpha internal consistency coefficient of the scale was obtained as 0.83 .

\subsection{Statistical Analysis}

In statistical analysis, the SPSS 23.00 package program was used. First, the Kolmogorov-Smirnov test was carried out to test whether the data were normally distributed. Since the data showed a normal distribution, independent samples t-test, one-way ANOVA, and LSD tests were used for comparison of variables.

\section{Findings}

The values obtained from the analyses conducted within the framework of the aim of the study are presented in tables.

\subsection{Anthropometric Features of Students}

Table 1 shows the comparison values of students with respect to gender in terms of anthropometric features. Accordingly, while there is no difference in the age variable, there are statistical differences in body height, body weight, and body mass index variables $(\mathrm{p}<0.01)$. The arithmetic mean of men is higher than that of women. 
Table-1. Comparison of anthropometric features of students in terms of gender variable.

\begin{tabular}{|c|c|c|c|c|c|}
\hline Variables & Categories & $\mathbf{N}$ & Mean & Sd & $\bar{t}$ \\
\hline \multirow{2}{*}{ Age } & Male & 230 & 22.82 & 1.90 & \multirow{2}{*}{1.25} \\
\hline & Female & 218 & 22.59 & 2.32 & \\
\hline \multirow{2}{*}{ Body height (cm) } & Male & 230 & 180.71 & 8.90 & \multirow{2}{*}{$5.63^{* *}$} \\
\hline & Female & 218 & 170.52 & 7.92 & \\
\hline \multirow{2}{*}{ Body weight $\left(\mathrm{kg} / \mathrm{m}^{2}\right)$} & Male & 230 & 77.50 & 15.02 & \multirow{2}{*}{$-11.39^{* *}$} \\
\hline & Female & 218 & 63.72 & 15.82 & \\
\hline \multirow{2}{*}{ Body mass index $\left(\mathrm{kg} / \mathrm{m}^{2}\right)$} & Male & 230 & 25.08 & 2.88 & \multirow{2}{*}{$-5.18^{* *}$} \\
\hline & Female & 218 & 23.38 & 3.29 & \\
\hline
\end{tabular}

Note: ${ }^{* *} \mathrm{p}<0.001$

Table 2 indicates that the arithmetic means of male and female students are close to each other and that the social appearance anxiety of the students is not different in terms of gender $(\mathrm{p}>0.05)$.

Table-2. Comparison of social appearance anxiety of students in terms of gender variable.

\begin{tabular}{c|c|c|c|c|c}
\hline Variables & Categories & N & Mean & Sd & t \\
\hline \multirow{2}{*}{ Gender } & Male & 230 & 34.43 & 10.57 & \multirow{2}{*}{-0.83} \\
\cline { 2 - 5 } & Female & 218 & 35.11 & 11.61 & \\
\hline
\end{tabular}

Table 3 shows that the social appearance anxiety of students is a difference in terms of the sports education status variable. Accordingly, it is seen that the social appearance anxiety of the students of the faculty of sport sciences is lower than the students of the faculty of education $(\mathrm{p}<0.01)$.

\begin{tabular}{c|c|c|c|c|c}
\multicolumn{2}{c}{ Table-3. Comparison of social appearance anxiety of students in terms of sports education status variable. } \\
\hline Variables & Categories & N & Mean & Sd & t \\
\hline \multirow{2}{*}{ Sports education status } & Faculty of Sport Sciences & 220 & 32.89 & 9.54 & \multirow{2}{*}{$-4.68^{* *}$} \\
\cline { 2 - 6 } & Faculty of Education & 228 & 36.68 & 12.19 & \\
\hline Note: ${ }^{* *} \mathrm{p}<0.001$.
\end{tabular}

When the comparison of students' social appearance anxiety in terms of body weight and height variables is examined, it is seen that students who are satisfied with these features are lower than those who do not Table 4 .

Table-4. Comparison of social appearance anxiety of students in terms of body weight and height variables.

\begin{tabular}{c|c|c|c|c|c}
\hline Variables & Categories & N & Mean & Sd & F/LSD \\
\hline \multirow{3}{*}{ Body weight $\left(\mathrm{kg} / \mathrm{m}^{2}\right)$} & Satisfied & 172 & 32.96 & 10.02 & \multirow{2}{*}{$13.30^{* *}$} \\
& Undecided & 66 & 35.22 & 10.12 & $1<3$ \\
\cline { 2 - 5 } & Unsatisfied & 210 & 37.48 & 9.00 & \\
\cline { 2 - 5 } & Satisfied & 172 & 33.43 & 9.60 & $11.68^{* *}$ \\
1 & Undecided & 66 & 38.44 & 8.73 & $1<2.3$ \\
\cline { 2 - 5 } & Unsatisfied & 210 & 37.02 & 9.65 & \\
\cline { 2 - 5 } & & &
\end{tabular}

Note: ${ }^{* *} \mathrm{p}<0.001$

\section{Conclusion}

This study was carried out to examine the social appearance anxiety of the faculty of sports sciences and faculty of education in terms of some variables. For this purpose, the results of the study are discussed with the results of other research.

The average age of the students participating in our study is 22.82 years for men and 22.59 years for women. It is seen that there is a significant difference in students' body weight, height, and body mass index values in terms of gender $(\mathrm{p}<0.01)$. However, body mass index values are within normal limits. It can be said that students receiving sports education increase their body mass index average. Body mass index value is considered to be normal between 20-25, 22 for men and 21 for women. In addition, the +1 high value of athletes is considered ideal (Çinar, Bostancı, İmamoğlu, Kabadayı, \& Şahan, 2004; Imamoğlu, Satıcı, Ermiş, \& Ermiş, 2018). Tekeli (2017) in his research, observed that the social appearance anxiety of physical education and sports education students is higher than those studying in other departments. Imamoğlu et al. (2018) in their research, found that the social appearance anxiety of the students who take sports education and do active sports is lower than the students who do not receive sports education and do not do active sports. Yıldırım, Cirak, and Konan (2011) in their research, found that there were no significant differences in the levels of social anxiety among pre-service teachers studying in different departments. When studies that are not parallel to these results are examined, different findings are observed. Alemdağ and Öncu (2015) found that pre-service teachers' social appearance anxiety in physical education and sports education department is lower than those studying in other departments. Doğan (2009) reported that men's social appearance anxiety is higher than women. Eren (2002) stated that social anxiety does not differ significantly by gender. Soylu, Atik, and Öçalan (2017) in their study on adolescents, observed that men's social appearance anxiety is higher than women. However, Çepikkurt and Çoşkun (2010) found that women, besides being less satisfied with their physical appearance in general, have negative feelings and thoughts about physical appearance compared to men. Villiers (2009) and Smits, Powers, Buxkamper, and Telch (2006) in their research, found that women experience higher social anxiety compared to men. Apart from these results, the research conducted in Turkey, anxiety of men is seen in the results of social anxiety is high compared to women (Alemdağ \& Öncu, 2015). 
Imamoğlu et al. (2018) observed no significant difference between social appearance anxiety and gender variable but found the average score of men high.

According to the results of our study, the social appearance anxiety point average in terms of gender was seen as 34.43 in women and 35.11 in men, but these values did not create a significant difference $(p>0.05)$. In our study, the social appearance anxiety scale score was found to be 32.89 in sports faculty students and 36.68 in education faculty students. It was determined that there was a significant difference between the students of the sports faculty (the students who received sports training) and the students of the faculty of education (students who did not receive sports training) $(\mathrm{p}<0.001)$. Students who receiving sports education gain many positive features such as competitions they attend, social environment interactions, meeting different cultures, and positive communication with the sense of self-confidence that sports bring to the person. Therefore, it is thought that the students of the faculty of sport sciences who receive sports education can be effective in keeping their social appearance anxieties lower than the students of the faculty of education since they transfer many positive features in sports life to their daily lives. It is known that participation in exercise has a positive effect on individuals' social appearance anxiety (Edwards, Ngcobo, Edwards, \& Palavar, 2005; Marquez \& McAuley, 2001). Yüceant (2013) found that the social appearance anxiety of pre-service teachers of physical education and sports is lower than the other departments. It is stated that sports have a positive effect on individuals in many areas from personality development to free time evaluation Koca, İmamoğlu, and İmamoğlu (2018). Regular physical activity and a healthy lifestyle have an important effect on the health of individuals, especially contributing to them to feel better and maintain their weight (Aslan \& Ziyagil, 2020; Atan, Tural, Imamoglu, Cicek, \& Tural, 2012). Research in the literature suggests that individuals who do sports have a much more friendly, extroverted, more patient, better at establishing social relations, better adapting to a new position, and a more psychologically balanced personality than individuals who do not do sports (Yamak \& İmamoğlu, 2019). The satisfaction of individuals with their body appearance may cause them to think positively (Yamak, İmamoğlu, İslamoğlu, \& Cebi, 2018). According to Tekeli (2017) the ones who have the highest level of social appearance anxiety among the students who receive physical sports training are those with low body weight. In addition, among the students at the faculty of education, those who have the highest social appearance anxiety are those with low body weight. According to the results of our study, the average of the students who are satisfied with their body weight is 32,96, and those who are not satisfied are 37,48. In addition, the average of those who were satisfied with their height was 33.43, while those who were not satisfied were 37.02. As a result, it was observed that the social appearance anxiety point averages of the students who were satisfied with their body weight and height were lower than the students who were not satisfied, and it was found to make a significant difference $(\mathrm{p}<0.001)$. Social appearance anxiety can be reduced by reaching the ideal weight in terms of body weight, and by psychological motivation in terms of height.

In summary, the most important result of our study is that the social appearance anxiety of the students who receive sports education is lower than the students who do not receive sports education. Based on this result, social appearance anxiety can be reduced by ensuring that students who do not receive sports training participate in sports activities.

\section{References}

Aksoy, Y., Aslan, H., \& İmamoğlu, O. (2020). Comparison of attention levels according to sport status and age category in students. Turkish Studies Educational Sciences, 15(3), 1473-1483.

Alemdağ, S., \& Öncu, E. (2015). Examination of pre-service teachers' concerns about participation in physical activity and social appearance. International Journal of Science Culture and Sport, 3(Special Issue 3), 287-300.

Aslan, H., \& Ziyagil, M. A. (2020). Relationships between body mass index and skinfold thickness of exercised and sedentary young adults. African Educational Research Journal, 8(3), 422-431. Available at: https://doi.org/10.30918/aerj.83.20.073.

Atan, T., Tural, E., Imamoglu, O., Cicek, G., \& Tural, S. (2012). Physical activity levels of teachers and health professionals in Turkey. Health Med, 6(6), 1935-1942.

Çepikkurt, F., \& Çoşkun, F. (2010). The levels of university dancers 'social physical anxiety and body imagment. Pamukkale Journal of Sport Sciences, $1(2), 17-24$.

Çınar, V., Bostancı, Ö., İmamoğlu, O., Kabadayı, M., \& Şahan, H. (2004). Anthropometric differences in student athletes according to branch and gender. Atatürk University Journal of Physical Education and Sport Sciences, Erzurum, 6(2), 26-34.

Dindar, M., \& Akbulut, Y. (2015). Role of self-efficacy and social appearance anxiety on gaming motivations of MMOFPS players. Computers E Education, 81, 26-34. Available at: https://doi.org/10.1016/j.compedu.2014.09.007.

Doğan, T. (2009). Examination of cognitive and self-evaluation processes in terms of social anxiety. Unpublished Doctoral Thesis. Sakarya University Institute of Social Sciences, Sakarya.

Edwards, S. D., Ngcobo, H. S., Edwards, D. J., \& Palavar, K. (2005). Exploring the relationship between physical activity, psychological wellbeing and physical self-perception in different exercise groups. South African Journal for Research in Sport, Physical Education and Recreation, 27(1), 59-74.

Eren, G. A. (2002). The effect of coping with social anxiety program on social anxiety levels of university students. Unpublished Doctoral Thesis. Ankara University Institute of Educational Sciences.

Ergür, E. (1996). The relationship between body-self perception and mental health in university students. Unpublished Master Thesis. Ege University Institute of Social Sciences, İzmir.

Gür, H., \& Küçükoğlu, S. (1992). Aging and physical activity (pp. 8). Istanbul: Roche Publications.

Hart, T. A., Flora, D. B., Palyo, S. A., Fresco, D. M., Holle, C., \& Heimberg, R. G. (2008). Development and examination of the social appearance anxiety scale. Assessment, 15(1), 48-59. Available at: https://doi.org/10.1177/1073191107306673.

Imamoğlu, G., Satıcı, A., Ermiş, E., \& Ermiş, A. (2018). Social appearance anxiety in students of the faculties of sport sciences. Paper presented at the Fine Arts and Education, 6th International Conference on Science Culture and Sport Using sports, culture, and social studies as means to rediscover lost values Proceedings Book.

Kılıç, M. (2015). Investigation of the relationship between university students' social appearance anxiety, self-esteem and loneliness levels. Master Thesis Selcuk University Institute of Health Sciences. Konya.

Koca, F., İmamoğlu, G., \& İmamoğlu, O. (2018). Sports status of high school students and investigation of personality characteristics by gender. The Journal of Academic Social Science, 6(80), 31-42.

Marquez, D. X., \& McAuley, E. (2001). Physique anxiety and self-efficacy influences on perceptions of physical evaluation. Social Behavior and Personality: An International Journal, 29(7), 649-659.

Mülazımoğlu, B. Ö., Erturan, İ., G., \& Arslan, Y. (2014). Achievement goals in Turkish high school PE setting: The predicting role of social physique anxiety. International Journal of Educational Research, 67, 30-39. Available at: https://doi.org/10.1016/j.ijer.2014.04.004. 
Smits, J. A., Powers, M. B., Buxkamper, R., \& Telch, M. J. (2006). The efficacy of videotape feedback for enhancing the effects of exposurebased treatment for social anxiety disorder: a controlled investigation. Behaviour Research and Therapy, 44(12), 1773-1785. Available at: https://doi.org/10.1016/j.brat.2006.01.001.

Soylu, Y., Atik, F., \& Öçalan, M. (2017). Investigation of adolescents' social appearance anxiety levels. Journal of Sports and Educational Sciences, $S I(1), 38-45$.

Tekeli, Ş. C. (2017). The comparison of social appearance anxiety and academic self-efficacy levels of physical education and sports teacher candidates and other teacher candidates. Bartın University Institute of Educational Sciences Physical Education and Sports Teaching Department Physical Education and Sports Teaching Department Master's Thesis.

Villiers, D. P. (2009). Perfectionism and social anxiety among college students. Doctoral Dissertation, The Department of Counseling and Applied Educational Psychology. Northeastern University Boston, Massachusetts.

Yamak, B., \& İmamoğlu, O. (2019). The beck hopelessness level according to behavior change stages in university Ondokuz Mayis students. Turkish Studies Educational Sciences, 14(3), 937-947.

Yamak, B., İmamoğlu, O., İslamoğlu, İ., \& Cebi, M. (2018). The effects of exercise on body posture. Turkish Studies Social Sciences, 13(18), $1377-1388$.

Yıldırım, T., Çırak, Y., \& Konan, N. (2011). Social anxiety in prospective teachers. İnönü University Journal of Education Faculty, 12(1), 85-100.

Yilldiran, İ., \& Yetim, A. (1996). A research on the priority aims of physical education course in secondary education. Gazi Journal of Physical Education and Sport Sciences, 1(3), 36-43.

Yüceant, M. (2013). Investigation of social appearance anxiety levels of physical education teacher candidates in terms of different variables. Master Thesis. Aksaray University Institute of Social Sciences. Aksaray. 